\title{
The Fermilab Neutrino Beam Program
}

\author{
R. A. Rameika \\ Fermi National Accelerator Laboratory, Batavia, Illinois 60506
}

\begin{abstract}
This talk presents an overview of the Fermilab Neutrino Beam Program. Results from completed experiments as well as the status and outlook for current experiments is given. Emphasis is given to current activities towards planning for a future program.
\end{abstract}

PACS: $14.60 . \mathrm{Lm}, 14.60 . \mathrm{St}$

\section{INTRODUCTION}

The past decade has been an active and exciting time for the Fermilab Neutrino Program. The observation of the atmospheric neutrino deficit was a smoking gun that the neutrinos may indeed be more interesting and complex than initially thought. The use of accelerator produced neutrinos to study neutrino properties have the advantage that the beams can be designed to control the neutrino flavor, limit the background and set a well defined direction and distance to the detector. On the other hand, hadron production models, which are used to predict neutrino fluxes are not that well constrained. This, in addition to poorly measured cross sections in the low energy region make all of these experiments quite challenging.

Nevertheless, in the mid to late 90 's the Fermilab Tevatron program used $800 \mathrm{GeV}$ protons to produce neutrinos for the NuTeV [1] and DONUT[2] experiments. NuTeV used a sign selected neutrino beam to measure the weak mixing angle, while the DONUT experiment made the first direct observation of tau neutrino interactions using a prompt neutrino beam produced in a tungsten beam dump. The late 90's also saw the beginning of planning for what is now the current program of the MINOS [3] and MiniBooNE [4] experiments. The MINOS experiment uses a conventional neutrino beam produced by $120 \mathrm{GeV}$ protons extracted from the Main Injector to the NuMI neutrino beamline, while the MiniBooNE neutrinos are produced by $8 \mathrm{GeV}$ protons from the Fermilab booster machine. The future neutrino program includes new experiments in the Booster Neutrino Beam (SciBooNE) [5] and the NuMI beam (Minerva [6] and NOvA [7]). Studies are also underway for much longer term planning which include conceptual designs of very large detectors located at very long baselines[8].

\section{THE DONUT EXPERIMENT}

The goal of the DONUT Experiment was to make direct observation of tau neutrino interactions by observing the production and subsequent decay of the tau lepton, in a prompt neutrino beam composed of muon, electron and tau neutrinos. The predicted composition of the prompt neutrino beam was approximately $4 \% v_{\tau}$, and the remainder equally split between $v_{\mu}$ and $v_{e}$. An emulsion target located 36 meters downstream of the beam dump was used as both the target and detector for the neutrino interactions. The experiment collected data in 1996-97. A total of 3.54 $\times 10^{17}$ protons on targed produced a total of $6.6 \times 10^{6}$ recorded triggers. The data reduction from triggers to tau candidates is summarized in Table 1. Two candidate events are shown in Figure 1. The experiment reported on the first four candidates in 2001[9] and the final results, including a measure of the $v_{\tau}$ cross sesction will be published in the near future.

\section{THE NUMI-MINOS PROJECT}

Construction of the NuMI/MINOS Project began in 1998 and was completed in 2004. The experiment began operations in March 2005 and anticipates operating until 2010. A schematic drawing of the NuMI beam components are shown in Figure 2. Details of the beam and detector were reported separately at this meeting [10]. Since this meeting, the 
TABLE 1. DONUT data reduction summary

\begin{tabular}{|c|c|}
\hline predicted vertices in the spectrometer & 1026 \\
\hline verticies in the fiducial volume & 812 \\
\hline digitized emulsion data exists & 633 \\
\hline emulsion vertex attempted & 633 \\
\hline vertex located & 581 \\
\hline systematic decay search & 563 \\
\hline$v_{\tau}$ candidates & 9 \\
\hline charm candidates & 7 \\
\hline
\end{tabular}
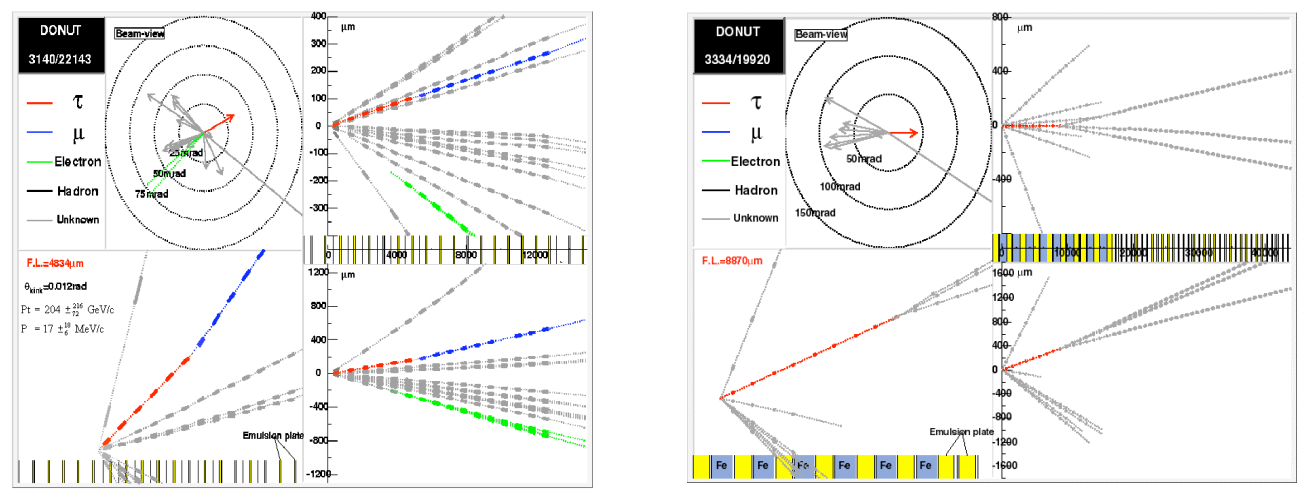

FIGURE 1. Two $v_{\tau}$ candidate events; The left event is a $\tau \rightarrow \mu$ decay and the event on the right is a $\tau \rightarrow 3 h$ decay.

MINOS collaboration has published its first results for a total of $1.27 \times 10^{20}$ protons on target [11], and has integrated a total of $3 \times 10^{20}$ protons on target.

\section{THE MINIBOONE EXPERIMENT}

In 1995 the LSND experiment [12] published results indicating a $v_{\mu} \rightarrow v_{e} \Delta m^{2}$ in the $\mathrm{eV}^{2}$ range. Given the accepted range of the $\Delta \mathrm{m}^{2}$ from the solar and atmospheric neutrinos, the LSND result could only be accommodated by the addition of a sterile neutrino. Since such a conclusion would require multiple confirmation, the MiniBooNE experiment was constructed to confirm or refute the LSND signal. The challenge for the experiment was to search for a $v_{e}$ appearance in a $v_{\mu}$ beam at the $\sim 0.3 \%$ level. With a baseline of $500 \mathrm{~m}$ and and a mean neutrino energy of 500 $\mathrm{MeV}$, the $\mathrm{L} / \mathrm{E}$ is essentially the same as LSND while both $\mathrm{L}$ and $\mathrm{E}$ being larger by a factor of $\sim 10$. Construction began in 1998 and was completed in 2002. The experiment has been in operation since 2002, and has integrated a total of 5.7 $\times 10^{20}$ protons producing neutrinos and is currently running in the $\bar{v}$ mode. A status report on the current state of the experiment was presented separately at this meeting [13]. Since this meeting, the MiniBooNE oscillation results have been published [14]. 


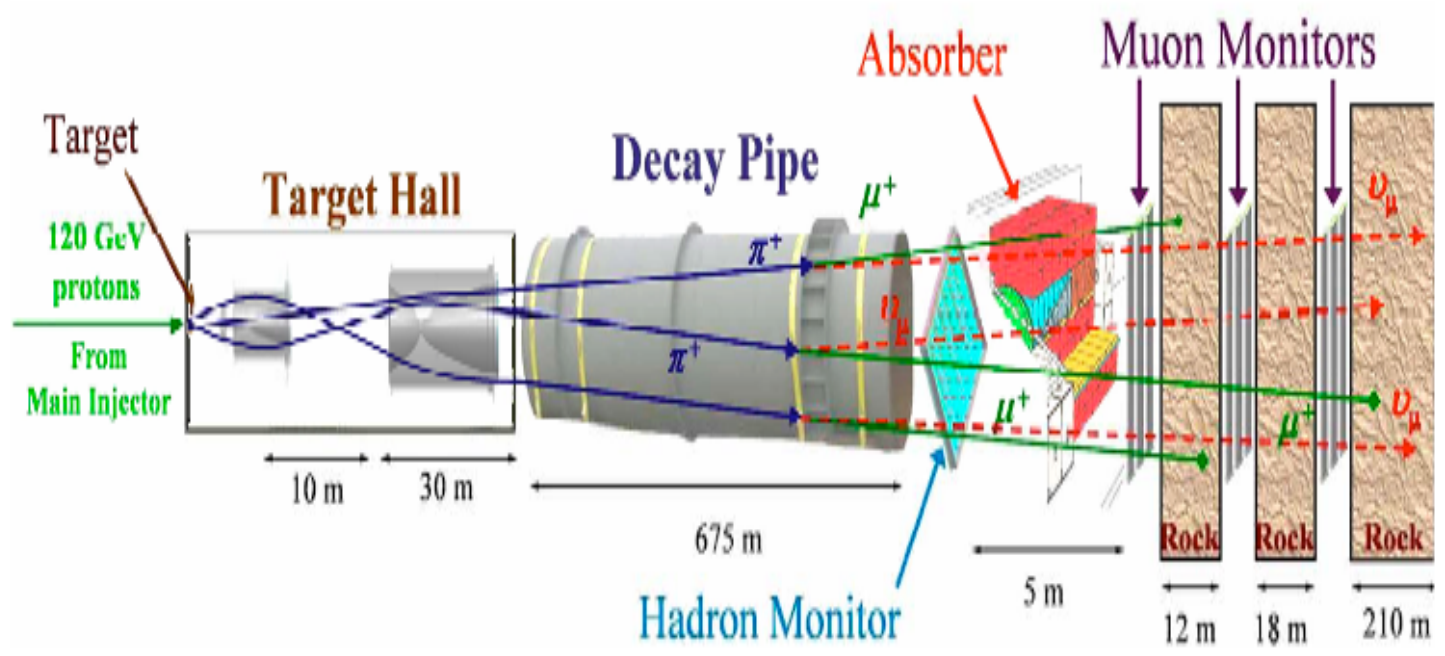

FIGURE 2. Schematic drawing of the NuMI beam components

\section{WHAT'S NEXT IN NEUTRINO PHYSICS?}

\section{$\theta_{13}$, Mass Hierarchy and $\delta_{C P}$}

The values of both the solar and atmospheric neutrino $\Delta m^{2}$ are relatively well established, as are the size of the mixing angles related to the primary oscillations $v_{\mu} \leftrightarrow v_{e}$ and $v_{\mu} \leftrightarrow v_{\tau}$. What is unknown is the strength of the mixing $v_{\mu} \rightarrow v_{e}$ which is the contribution of $v_{e}$ in the $v_{3}$ mass state. The mixing angle is referred to as $\theta_{13}$. In addition to not knowing the size of $\theta_{13}$, the relative ordering of the $v_{3}$ state with respect to the closely spaced $v_{1}$ and $v_{2}$ states. These unknowns are summarized pictorially in Figure 3. Additionally, an undetermined CP phase, $\delta$, is also one of the unknowns. In fact, a determination of a value of $\delta$ different from 0 and $\pi$, is a measure of CP violation in the neutrino sector. It turns out that neutrino beams traveling through the earth are affected by the matter in the earth. And infact, the magnitude of the effect depends on whether nature has configured the neutrino mass states in the normal or inverted hierarchy. In the normal hierarchy, the matter effect enhances the oscillation of neutrinos and suppresses the oscillation of anti-neutrinos. Conversely, for the inverted hierarchy, the anti-neutrino oscillations will be enhanced and the neutrino oscillations will be suppressed. One other feature of the matter effect is that it is an increasing function of energy. To complicate the situation, recall that $\mathrm{CP}$ violation will affect the oscillation probabilities of neutrinos and anti-neutrinos in opposite directions, so we find that there can be a confusion or ambiguity in determining how much each effect is contributing to the oscillation probability. A simple example to illustrate this confusion is shown in Figure 4. One can see from this example, that one possible way to resolve the ambiguity is to look at the 2nd oscillation maximum (at $0.8 \mathrm{GeV}$ ). At this energy, the matter effect is minimal, and hence any difference in the $v$ to $\bar{v}$ oscillation probability can be attributed to a $\mathrm{CP}$ effect.

\section{The NuMI Off-Axis Beam}

A nice feature of neutrino beams is that while the target and focusing horn configuration sets the energy spectrum of the neutrinos along the beam axis, the spectrum at an off-axis position is determined by the kinematics of the $\pi$ and $\mathrm{K}$ decays, and results in a narrow band spectrum. Figure 5 shown the NuMI off-axis spectrum at a location of $12 \mathrm{~km}$ off of the beam axis, at a distance of $810 \mathrm{~km}$. A detector placed at this location will be sitting very close to the peak 


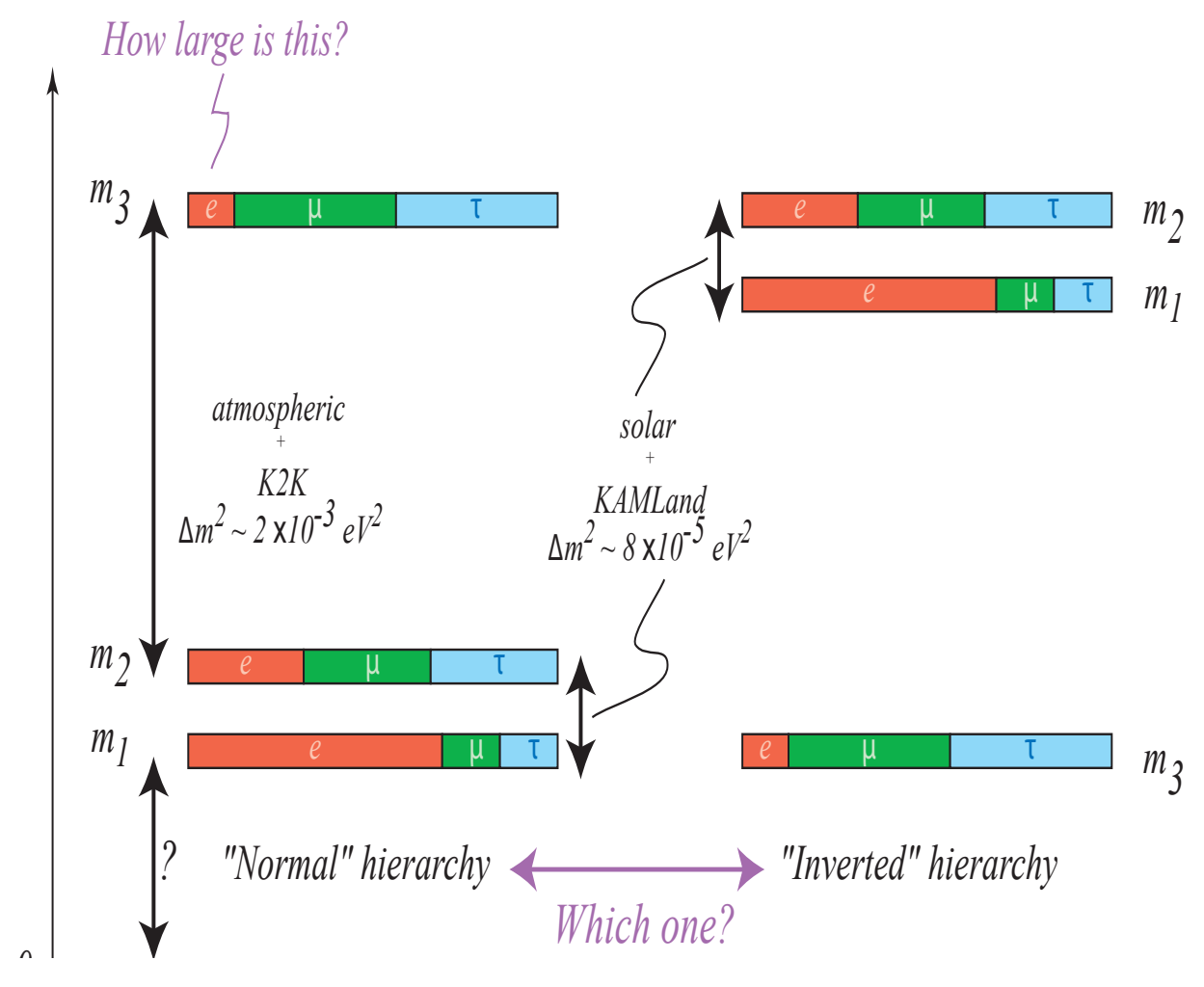

FIGURE 3. The open questions in neutrino physics are the amount of $v_{e}$ in the $v_{3}$ mass state and the mass ordering of the states.

of the first oscillation maximum. The NOvA experiment will construct a massive (15- 18 kton) liquid scintillator detector at this location, which is in Ash River Minnesota. The goal of the NOvA experiment is to achieve sensitivity to $\sin ^{2} 2 \theta_{13}$ down to $\sim 0.01$. Sensitivity to the mass hierarchy is possible if $\sin ^{2} 2 \theta_{13}$ is shown to be close to the current limit of $\sim 0.015$ set by the Chooz experiment [15]. Accelerator upgrades are included in the NOvA project to improve the proton intensity delivered to the NuMI beam from the current level of $\sim 300 \mathrm{~kW}$ to $\sim 700 \mathrm{~kW}$.

Figure 6 shows a schematic of the NuMI beam spectrum at the first and second oscillation maximum, with the oscillation probability (no matter or CP effects) overlaid. While this illustrates that a two detector experiment, one at the first maximum, and one at the second, might be a way to resolve ambiguities, it can be seen that the event rate at the second maximum is very low, compared to the rate at the first. The factor is approximately 20 , which would imply that one needs for the second detector to be very, very massive in order to have any sensible statistics. In the course of the Future Long Baseline study, several scenarios of detector mass and exposure were studied to determine the sensitivity of a long term off-axis program using the NuMI beam [8]. 

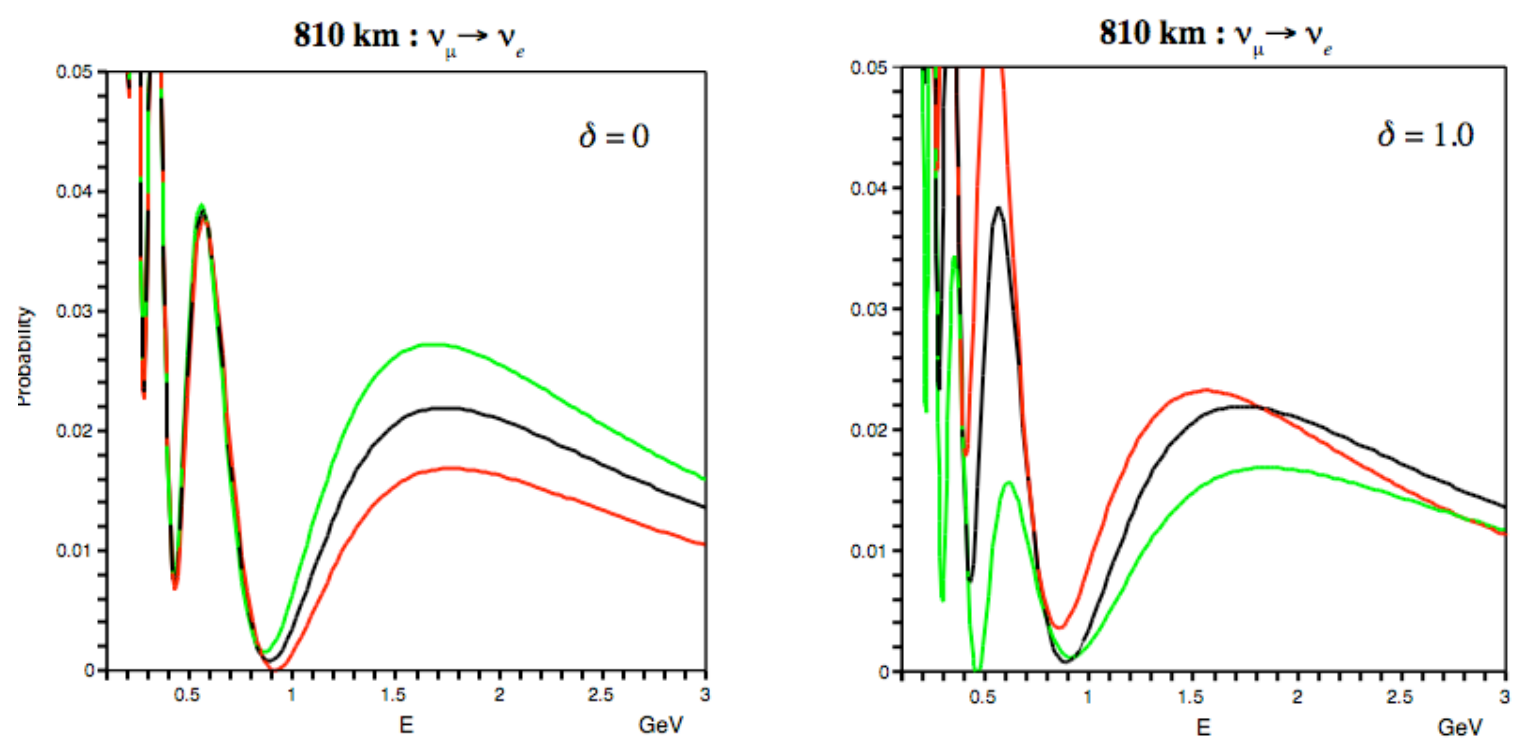

FIGURE 4. Oscilation probabilities with matter effect $\left(\delta_{C P}=0\right)$ and $C P$ effect $\left(\delta_{C P}=1.0\right.$ and matter effect $\left.=0\right)$. These plots are for a normal mass ordering and $\sin ^{2} 2 \theta_{13}=0.04$.

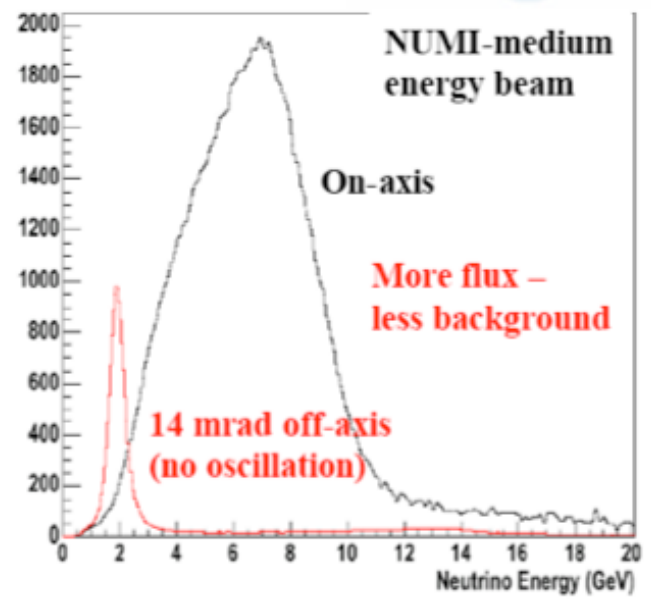

FIGURE 5. The NuMI Off-axis spectra at a baseline of $810 \mathrm{~km}$ and $12 \mathrm{~km}$ off-axis.

Wide Band Beam to DUSEL

An alternative to the off-axis approach is to place the detectors on (or near) the beam axis. The energy spectrum of the wide band beam can be tuned such that it covers both the first and second oscillation maximum. This appears to be 


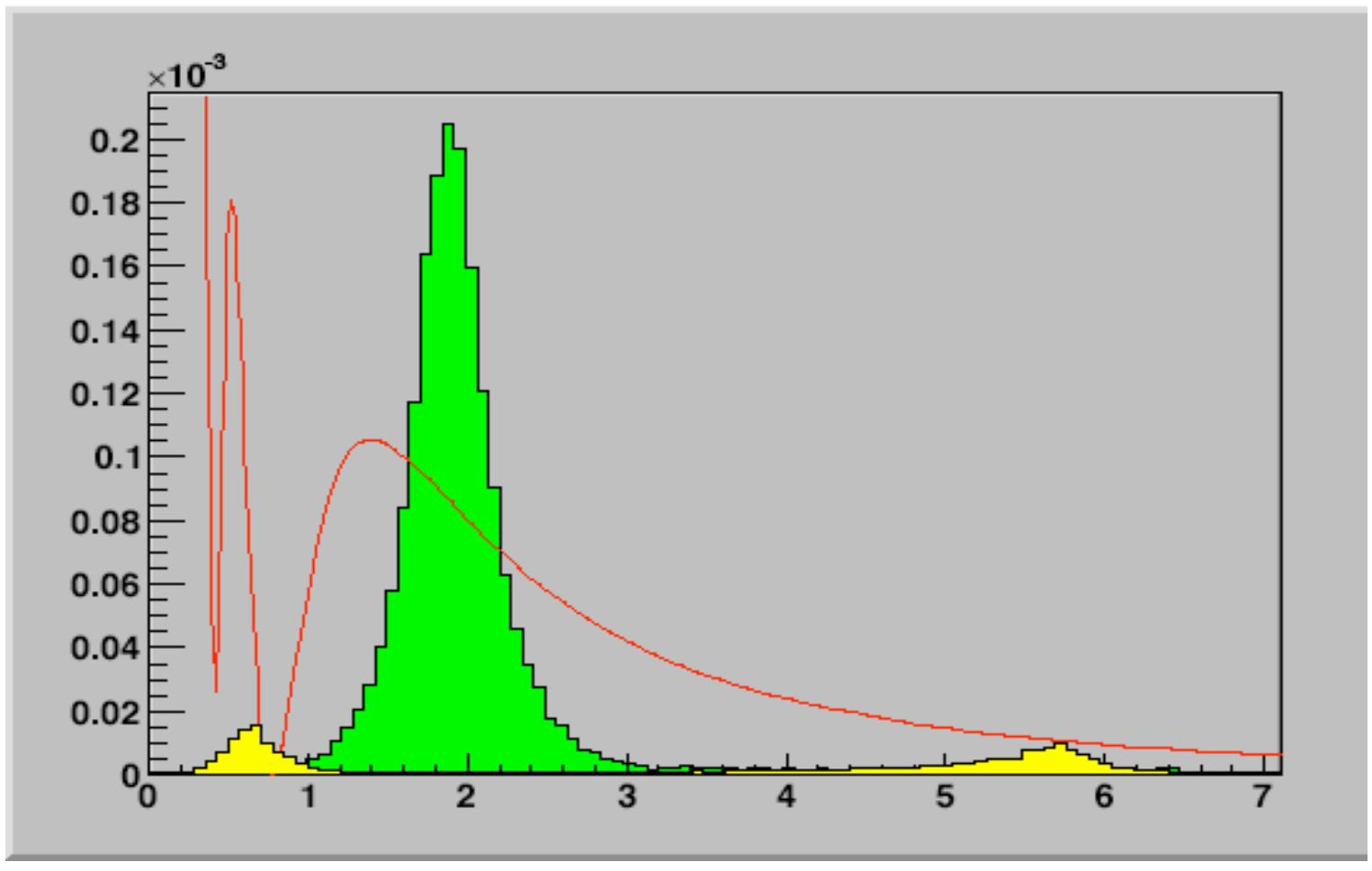

FIGURE 6. The NuMI Off-axis spectra at a baseline of $810 \mathrm{~km}$ at $12 \mathrm{~km}$ (14 mrad) off-axis and $32 \mathrm{~km}(40 \mathrm{mrad})$ off-axis.The oscillation probability (with out matter or $\mathrm{CP}$ effects) is overlayed.

an optimum configuration for sensitivity to both the mass hierarchy and $\delta_{C P}$. A new beamline from Fermilab aimed at the proposed DUSEL [16] site at the Homestake Mine in South Dakota would have a baseline of $\sim 1300 \mathrm{~km}$. A very massive detector (or detectors) placed deep underground would also be used to search for proton decay.

\section{Proton Plans and Large Detectors}

Regardless of the configuration chosen, wide band or off-axis, to achieve sensitivity to the mass hierarchy and CP violation down to the level of $\sin ^{2} 2 \theta_{13} \sim 0.01$ will require proton intensities $>1 \mathrm{MW}$, long running time and masses $>100$ ktons. There are two possible plans for achieving higher intensity from the Fermilab complex. The first option is an upgrade of the existing Main InJector/NuMI facility called SNuMI. SNuMI could produce $\sim 1.2 \mathrm{MW}$ at 120 $\mathrm{GeV}$. A second option, which has the potential to achieve proton power of $\sim 2.3 \mathrm{MW}$ at $120 \mathrm{GeV}$, is to build a new super conducting RF linac. This concept has been under consideration for several years. An early concept was called a "Proton Driver", and a revised concept was termed "HINS", for High Intensity Neutrino Source. Most recently (August 2007) the concept has been re-presented as "Project X". The timeline for realization of a high powered proton source is the middle of the next decade.

While waiting for protons, there are real challenges to solve in the detector area. Two technologies are viable candidates for very large ( $\geq 100$ ktons) detectors. Water cerenkov, like SuperK, is a proven technology to the 50 kton scale. The technical issue about going larger is mostly one cost and procurement of the large number of phototubes 
required. Experimentally, background rejection capability may become the limiting factor in such a detector's ultimate capability.

The alternative technology is a time projection chamber (TPC) in Liquid Argon. Liquid Argon has the potential to have $\sim 80 \%$ efficiency for electron identification and excellent background rejection. The problem is that this technology has yet to be really implemented even at a moderate scale, let alone the multi-kiloton scale. A staged $\mathrm{R} \& \mathrm{D}$ plan leading towards the development of a large scale detector has been proposed. A schematic showing the main elements of such a program is in Figure 7. The ultimate feasibility of detectors on the order of $\sim 100$ ktons will depend on the success of the R\&D.

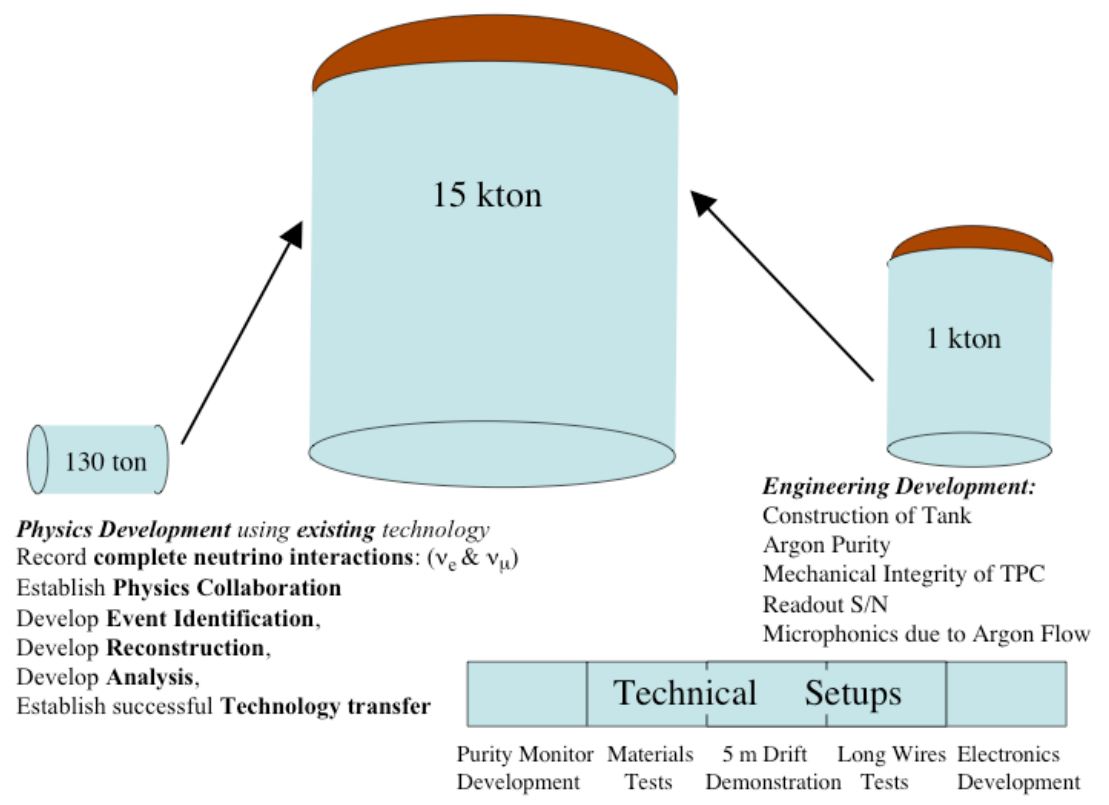

FIGURE 7. Schematic diagram of a staged Liquid Argon R\&D program.

\section{Neutrino Cross sections}

Several decades of experiments operating at high energy $(>10 \mathrm{GeV})$ have enabled neutrino cross sections at high energy to be well measured. In contrast, data in the energy regime of $\sim 1 \mathrm{GeV}$ is relatively sparse, and particularly for antineutrinos. This is a particular concern for upcoming oscillation experiments, namely T2K [17] and NOvA, since the energy region of interest is $0.5-\sim 3 \mathrm{GeV}$. Fermilab is currently planning two experiments dedicated to directly addressing the issue of neutrino cross sections. 
The SciBooNE experiment is located in the Booster Neutrino Beam, just $100 \mathrm{~m}$ downstream of the focusing horn. Because it is relatively close to the target, the detector can be rather small, and is in fact the SciBar detector that was used in the near location of the K2K [18] experiment. For approximately two years of data taking, the experiment hopes to collect $\sim 80 \mathrm{~K}$ events. The beam spectrum and the SciBooNE detector and comparison with the planned T2K spectrum is shown in Figure 8.

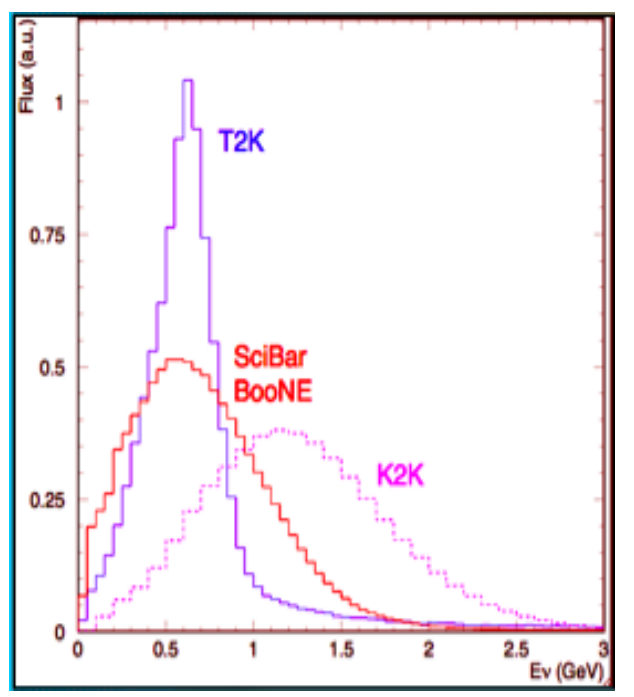

FIGURE 8. The spectra of the Booster Neutrino beam at the SciBooNE detector. It is compared to the expected spectra for the $\mathrm{T} 2 \mathrm{~K}$ experiment and the previously run $\mathrm{K} 2 \mathrm{~K}$ experiment.

\section{Minerva}

The Minerva Experiment will be installed just upstream of the MINOS near detector and will begin taking data in 2010, and continue to run through the NOvA era. The primary target in the Minerva detector is solid scintillator, making it an "active" target. Targets of iron and lead can also be installed so the the A-dependence of the cross sections can also be measured. Minerva expects to collect nearly 10 million events on the scintillator target, and a few million each on the other targets. Data will also be taken using the low, medium and high energy configurations of the NuMI beam, giving results over a broad energy range.

\section{PEANUT}

A small project taking advantage of the existing NuMI beam is the PEANUT test which is located just upstream of the MINOS near detector. This is a test exposure of emulsion ECC bricks of the type being installed in the OPERA detector at the CNGS [19]. Forty eight bricks, arranged in 4 walls are interleaved with the scintillating fiber tracker from the DONUT experiment. The goal of the test is to collect and reconstruct $v_{\mu}$ and $v_{e}$ neutrino interactions. Not only will this help in preparation for the OPERA data, but may also provide some valuable contribution to determining the $v_{e}$ content of the NuMI beam. 


\section{DEVELOPING A ROAD MAP}

Developing a road map for future neutrino experiments first depends on establishing that $\theta_{13}$ is actually measurable. In the next several years ( 2009-2012) results should be available from the reactor experiments, Double Chooz [20] and Daya Bay [21]. By 2012 first results should also be available from the JPARC-T2K experiment. From these experiments it should be determined whether or not $\sin ^{2} 2 \theta_{13}$ is greater than 0.03 . If it is smaller than this, continued running of the T2K experiment and the NOvA experiment, which should begin to come on line in 2012, will be able to push the sensitivity down to or below $\sin ^{2} 2 \theta_{13} \sim 0.01$. Of course if nature is kind, and $\sin ^{2} 2 \theta_{13}$ turns out to be near the current value of $\sim 0.1$, then the NOvA experiment, running both $v$ 's and $\bar{v}$ 's would have some probability of determining the mass hierarchy.

\section{CONCLUSIONS}

The Fermilab Neutrino Beam Program is active and exciting. After one year of operation, the MINOS experiment reported its first results and continues to operate efficiently. MiniBooNE results were reported in spring 2007. Construction of a new experiment, SciBooNE, in the Booster Neutrino beam has been completed and the experiment is taking data. The Minerva experiment is on track for a construction start in fiscal year 2008. Construction of the NOvA project is also scheduled to begin in 2008.

Planning for the future is challenging. The physics potential is great but difficult to achieve. Several options for beam configuration and detector technology exist, but in all cases, high proton intensity and very massive detectors are required. Support for R\&D in both the accelerator and detector areas is essential.

\section{REFERENCES}

1. NuTeV web site : http://www-e815.fnal.gov

2. DONUT web site : http://www-donut.fnal.gov

3. MINOS web site : http://www-numi.fnal.gov

4. MiniBooNE web site : http://www-boone.fnal.gov

5. SciBooNE web site : http://www-sciboone.fnal.gov

6. Minerva web site : http://minerva.fnal.gov

7. NOvA web site $:$ http://www-nova.fnal.gov

8. Long Baseline Study web site : http://nwg.phy.bnl.gob/fnal-bnl

9. Physics Letters B 504,218 (2001).

10. see talk by Alysia Marino

11. D. G. Michael et. al.,Phys. Rev. Lett. 97, 191801 (2006)

12. A web site with references : http://www.nu.to.infn.it/exp/all//snd

13. see talk by Eric Zimmerman

14. A. A. Aguilar-Arevalo, et. al., Phys. Rev. Lett. 98, 231801 (2007)

15. M. Apollonio et.al.,Eur. Phys. J. C 27, 331 (2003)

16. Deep Underground Science and Engineering Laboratory (DUSEL) web site : http://www.dusel.org

17. T2K web site : http://jnusrv01.kek.jp/public/t2k

18. K2K web site $:$ http://neutrino.kek.jp

19. Opera web site : http://operaweb.web.cern.ch/operaweb

20. Double Chooz web site : http://doublechooz.in2p3.fr

21. Daya Bay web site : http://dayawane.ihep.ac.cn 